\title{
Perioperative Factors Associated with Severe Pain in Post-Anesthesia Care Unit after Thoracolumbar Spine Surgery: A Retrospective Case-Control Study
}

\author{
Paweenus Rungwattanakit ${ }^{1}$, Tarnkamon Sondtiruk ${ }^{2}$, Akarin Nimmannit ${ }^{3}$, Busara Sirivanasandha $^{1}$ \\ ${ }^{1}$ Department of Anesthesiology, Faculty of Medicine Siriraj Hospital, Mahidol University, Bangkok, Thailand \\ ${ }^{2}$ Department of Nursing, Faculty of Medicine Siriraj Hospital, Mahidol University, Bangkok, Thailand \\ ${ }^{3}$ Department of Research, Faculty of Medicine Siriraj Hospital, Mahidol University, Bangkok, Thailand
}

Study Design: A retrospective case-control study.

Purpose: To evaluate the effect of nitrous oxide and anesthetic and operative factors associated with severe pain in the early postoperative period after thoracolumbar spine surgery.

Overview of Literature: Thoracolumbar spine surgery is the most common procedure in spine surgery, and up to $50 \%$ of the patients suffer from moderate to severe pain. Nitrous oxide has analgesic, anxiolytic, and anesthetic effects; nevertheless, its benefits for early postoperative pain control and opioid consumption remain to be established.

Methods: The medical records of eligible participants who underwent thoracolumbar spine surgery between July 2016 and February 2017 were reviewed. Enrolment was performed consecutively until reaching 90 patients for the case (severe pain) group (patients with a pain score of $\geq 7$ out of 10 at least once during the post-anesthesia care unit [PACU] admission), and 90 patients for the control (mild-to-moderate pain) group (patients with a pain score of $<7$ in every PACU assessment). The data collected comprised patient factors, anesthetic factors, surgical factors, PACU pain score, and PACU pain management.

Results: A total of 197 patients underwent thoracolumbar spine surgery with an incidence of early postoperative severe pain of $53.3 \%$. The case-control study revealed no differences in the factors related to pain intensity. A subgroup analysis was performed for failed back surgery syndrome (FBSS), spinal stenosis, and spondylolisthesis. After multivariate analyses, only the age group of 19-65 years and the baseline Oswestry Disability Index (ODI) were found to be significant risk factors for early postoperative severe pain in the PACU (odds ratio [OR], 2.86; 95\% confidence interval [CI], 1.32-6.25; OR, 1.03; 95\% Cl, 1.01-1.05, respectively).

Conclusions: Nitrous oxide, anesthetic agents, and surgical techniques did not affect the early postoperative pain severity. Age under 66 years and the baseline ODI were the significant risk factors for pain intensity during the early postoperative period of the FBSS, spinal stenosis, and spondylolisthesis subgroups.

Keywords: Postoperative pain; Nitrous oxide; Spine surgery; Patient-controlled analgesia; Oswestry Disability Index; Post-anesthesia care unit

Received May 28, 2018; Revised Sep 20, 2018; Accepted Oct 18, 2018

Corresponding author: Busara Sirivanasandha

Department of Anesthesiology, Faculty of Medicine Siriraj Hospital, Mahidol University, 2 Wanglang Road, Bangkok Noi, Bangkok 10700, Thailand

Tel: +66-2-419-7978, Fax: +66-2-411-3256, E-mail: busarasiri@gmail.com 


\section{Introduction}

Thoracolumbar spine surgery is one of the most common procedures performed in spine surgery. Up to $50 \%$ of the patients suffer from moderate to severe pain after the procedure [1,2], and, in a comparison of 179 surgical procedures, major spine surgery accounted for three of the top six surgeries with postoperative severe pain [3]. In two previous thoracolumbar spine surgery studies, the means of the pain scores by Visual Analog Scale (VAS) score were 7.8 out of 10 [4] and 6.8 out of 10 [5]. This pain score may increase the incidence of delirium [6] and the duration of post-anesthesia care unit (PACU), thereby pushing up the cost of care [7]. Factors identified as possibly affecting the degree of postoperative pain were depression, preoperative pain score, and chronic preoperative opioid use $[4,8,9]$.

Despite the increased awareness about the need for pain management, a 2014 Danish study concluded that pain management after spine surgery was still inadequate [2]. In addition, a survey conducted by a nurse anesthetist in Thailand in 2009 reported that postoperative pain control was undermanaged in most of the Thai hospitals [10]. For example, some hospitals did not record pain scores in the PACU or did not have postoperative pain management guidelines.

In the case of the Siriraj Hospital, for instance, about 300-400 thoracolumbar spinal surgeries are conducted in a year. Up to $50 \%$ of the patients in the PACU suffer from severe pain with a 0-10 Numerical Rating Scale (NRS) of $\geq 7$, which needs to be improved. Currently, pain management at the Siriraj Hospital is mainly achieved via the intermittent use of intravenous (IV) opioids. If patients are undergoing extensive spine surgery and are expected to have severe pain after surgery, an IV patient-controlled analgesia (PCA) is administered. Previous studies have shown that IV PCA is a better method of pain relief than intermittent IV opioids. Intrathecal morphine or continuous epidural analgesia provides better pain relief than an IV PCA, although the patients may experience the side effects of nausea, vomiting, and itching [5,11].

Nitrous oxide $\left(\mathrm{N}_{2} \mathrm{O}\right)$ is an anesthetic gas that has been used for more than 150 years. It has analgesic, anxiolytic, and anesthetic effects when used via the opioid-dependent mechanism and the gamma-aminobutyric acid and $\mathrm{N}$-methyl-D-aspartate receptors [12,13]; moreover, it also decreases pain hypersensitivity in neuropathic pain [13].
Nevertheless, the benefits of using $\mathrm{N}_{2} \mathrm{O}$ for the provision of early postoperative pain control and reduced opioid consumption remains to be established [14], and it may increase the risk for postoperative nausea and vomiting. Therefore, the use of $\mathrm{N}_{2} \mathrm{O}$ is largely dependent on the preferences of individual anesthesiologists. From our experience together with the data obtained from the routine clinical service at the Siriraj Hospital, the patients who underwent thoracolumbar spine surgery and were exposed to $\mathrm{N}_{2} \mathrm{O}$ during general anesthesia appeared to be more likely to develop severe pain (NRS pain $\geq 7$ ) in the PACU than if not exposed to $\mathrm{N}_{2} \mathrm{O}$. Postoperative pain control in spine patients would be improved if it could be confirmed that there are significant associations between the use of $\mathrm{N}_{2} \mathrm{O}$ and the incidence of severe pain in the PACU, and if the other factors that may be associated with pain (such as age, sex, intraoperative opioids, and type of surgery) could be identified.

\section{Materials and Methods}

\section{Study participants}

After this retrospective, case-control study had been approved by the Siriraj Institutional Review Board in May 2017 (Si 270/2017), a review was conducted of the medical records of eligible participants who had been admitted to the PACU, Siriraj Hospital. The recruitment was performed in a consecutive order of PACU admission post July 2017 until 90 patients were identified for each of the two following groups: a severe pain group and a mild-tomoderate pain group. Patients with a pain score of $\geq 7$ out of 10 on at least one occasion during their PACU admission were allocated to the severe-pain group, while those with a pain score of $<7$ in every assessment were assigned to the mild-to-moderate pain group.

The medical records were scrutinized by two reviewers as follows: a nurse anesthetist and a PACU nurse. During the reviewer training, the study protocol, definitions, and the case record form were explained. Initially, both the reviewers practiced completing the case record forms for 10 cases together. Next, each reviewer independently examined half of the remaining 170 medical records to prepare a form for each case. Each reviewer subsequently checked every 20th form completed by the other reviewer, and 60 of the total 180 case record forms were randomly checked by a third reviewer (an anesthesiologist), following which, 
all 180 forms were converted to electronic data for input to PASW SPSS Statistics for Windows ver. 18.0 (SPSS Inc., Chicago, IL, USA). The data comprised the following: age; sex; American Society of Anesthesia (ASA) classification; preoperative pain score; baseline Oswestry Disability Index (ODI); history of chronic pain; surgical diagnosis; type of operation; level of spine surgery; surgical duration; bleeding; intraoperative opioid consumption; use of $\mathrm{N}_{2} \mathrm{O}$ and inhalation; local anesthetic infiltration; pain score; duration and degree of opioid consumption in the PACU; and use of PCA.

\section{Statistical analysis}

The calculation of the sample size was based on the pilot study finding that the incidence of severe pain after thoracolumbar spine surgery at the Siriraj Hospital's PACU was approximately $50 \%$. It was thus assumed that the incidence of early postoperative severe pain after thoracolumbar spine surgery would be greater among patients who received $\mathrm{N}_{2} \mathrm{O}$ during general anesthesia than among those who did not. To detect a clinically significant difference with a power of $80 \%$, an alpha error of 0.05 (twosided) and an odds ratio (OR) of 2.5, each group required 80 patients. To compensate for data that may be found to be missing during the retrospective review, the sample size was adjusted upward to 90 cases for each group.

The data analysis was performed using the independent sample Student $t$-test, the Pearson's chi-square test, and the Mann-Whitney $U$-test, and it employed the program PASW SPSS Statistics for Windows ver. 18.0 (SPSS Inc.). All descriptive data were presented as case (\%), mean \pm standard deviation, and median (interquartile range). Univariate analyses of each factor were performed to calculate the $p$-value, OR, and $95 \%$ confidence interval (CI). Multiple logistic regression analysis was performed by choosing the factors with a $p$-value of $<0.10 ; p<0.05$ was considered to be significant.

\section{Results}

Of the 197 patients who underwent thoracolumbar spine

Table 1. Demographic data

\begin{tabular}{|c|c|c|c|}
\hline \multirow{2}{*}{ Characteristic } & \multicolumn{2}{|c|}{ Pain intensity in PACU } & \multirow{2}{*}{$p$-value } \\
\hline & Mild-moderate pain $(n=90)$ & Severe pain $(n=90)$ & \\
\hline Age (yr) & $58.6 \pm 18.7$ & $58.2 \pm 13.7$ & 0.849 \\
\hline Sex (female) & $53(58.9)$ & $53(58.9)$ & 1.000 \\
\hline Body mass index $\left(\mathrm{kg} / \mathrm{m}^{2}\right)$ & $25.2 \pm 5.36$ & $24.6 \pm 4.03$ & 0.423 \\
\hline American Society of Anesthesia status & & & 0.106 \\
\hline I & $8(8.9)$ & $16(17.8)$ & \\
\hline$\|$ & $56(62.2)$ & $57(63.3)$ & \\
\hline III & $26(28.9)$ & $17(18.9)$ & \\
\hline Preoperative pain score (Visual Analog Scale) & $4.1 \pm 2.8$ & $4.7 \pm 2.5$ & 0.105 \\
\hline Baseline Oswestry Disability Index (missing 19 cases) & $43.9 \pm 20.5$ & $49.4 \pm 17.8$ & 0.070 \\
\hline Chronic pain & $45(50.0)$ & $47(52.2)$ & 0.882 \\
\hline Surgical diagnosis & & & 0.699 \\
\hline Failed back surgery syndrome & $8(8.9)$ & $9(10.0)$ & \\
\hline Adolescent idiopathic scoliosis & $5(5.6)$ & $1(1.1)$ & \\
\hline Spinal stenosis & $38(42.2)$ & $41(45.6)$ & \\
\hline Spondylolisthesis & $25(27.8)$ & $27(30.0)$ & \\
\hline Fracture/cancer/infection & $11(12.2)$ & $9(10.0)$ & \\
\hline Others & $3(3.3)$ & $3(3.3)$ & \\
\hline Numerical Rating Scale in PACU & $0.59 \pm 1.3$ & $8.5 \pm 1.2$ & $<0.001$ \\
\hline
\end{tabular}

Values are presented as mean \pm standard deviation or number (\%).

PACU, post-anesthetic care unit. 
surgery during July 2016-February 2017, the incidence of early postoperative severe pain was $53.3 \%$. The medical records were reviewed to identify 180 cases for inclusion in the study. Three cases were dropped completely because important data were missing. Ninety cases were subsequently selected for the mild-to-moderate pain group, and 90 others for the severe-pain group. Both groups were similar in terms of age, sex, body mass index, ASA status, preoperative pain score, baseline ODI, history of chronic pain, and surgical diagnosis (Table 1). Only one patient was diagnosed with depression, and three others were on amitriptyline or nortriptyline. One patient was recorded taking an antiepileptic drug.

$\mathrm{N}_{2} \mathrm{O}$ was used in 59 cases, which included 29 out of the 90 cases (32.2\%) in the mild-to-moderate pain group and 30 out of the 90 cases (33.3\%) in the severe-pain group $(p=1.00)$. The prevalence of using $\mathrm{N}_{2} \mathrm{O}$ was not statistically different for the early postoperative mild-to-moderate and severe-pain patient groups.

Univariable and multivariable analyses were performed by selecting the factors whose $p$-values were $<0.10$, namely, the age-group, baseline ODI, surgical duration, blood loss, total morphine consumption, and IV PCA-opioid use. Only the IV PCA-opioid use was found to be a marginally significant preventive factor for early postoperative severe pain in the PACU ( $p=0.05$; OR, 0.05 ; 95\% CI, $0.24-1.00)$.

To understand the subject better, a post hoc analysis was performed in the subgroup of patients whose diagnoses suggested that they were prone to high postoperative pain scores. These diagnoses were failed back surgery syndrome (FBSS), spinal stenosis, and spondylolisthesis (Table 2). They also formed the majority of patients in our setting $(\mathrm{n}=148)$.

The proportion of patients who experienced severe pain in the PACU tended to be higher in the 19-65-year age group than in the $>65$-year age group $(p=0.056)$. The mean baseline ODI was significantly higher for the severepain group than for the mild-to-moderate pain group, with scores of $49.7 \pm 16.2$ and $42.2 \pm 20.6$, respectively $(p=0.018)$. The mean surgical duration was significantly longer for the mild-to-moderate pain group $(242 \pm 108$ minutes) than for the severe-pain group (204 \pm 76 minutes, $p=0.018$ ). The intraoperative blood loss was greater in the mild-to-moderate pain group than in the severe-pain group, with median (interquartile range) blood losses of $500 \mathrm{~mL}$ (range, $250-800 \mathrm{~mL}$ ) and $400 \mathrm{~mL}$ (range, 175-700
$\mathrm{mL}$ ), respectively $(p=0.055)$. The intraoperative morphine equivalences did not differ between the two groups. More patients in the mild-to-moderate pain group received IV PCA-opioids than in the severe-pain group, with 25 cases (35.2\%) versus 15 cases (19.5\%), respectively, and $p=0.031$.

The multiple logistic regression analysis was performed by selecting the factors whose $p$-values were $<0.10$, namely the age group, baseline ODI, surgical duration, blood loss, and IV PCA-opioid use (Table 3). Only the 19-65-year age group and baseline ODI were found to be significant risk factors for early postoperative severe pain in PACU. The age group 19-65 years had a $p=0.007$ and an adjusted OR of 2.86 (95\% CI, 1.32-6.25). The baseline ODI had $p=0.004$ and an adjusted OR of 1.03 (95\% CI, 1.01-1.05).

The postoperative results are shown in Table 4 . The PACU duration for the severe-pain group (86.2 \pm 27.3 minutes) was longer than that for the mild-to-moderate pain group (78.4 \pm 25.4 minutes, $p=0.048)$. Morphine consumption during PACU admission was also higher for the severe-pain group than for the mild-to-moderate pain group (the median consumptions were 4 and $0 \mathrm{mg}$, respectively; $p<0.001)$. Moreover, the frequency of opioids received in the PACU was higher for the severe-pain group (2.5 times) than for the mild-to-moderate group (0 times, $p<0.001)$.

In addition, the pain intensity during the 24-hour period after surgery was higher for the severe-pain group. The median of the highest VAS pain score at 0-6 hours was 5 for the severe-pain group as compared with 4 for the mild-to-moderate group ( $p=0.001)$. At 7-24 hours, the scores for the two groups were 4 and 2 , respectively $(p=0.002)$.

Furthermore, the incidences of pain in the severepain and mild-to-moderate groups at 0-6 hours were 33 (37.1\%) and 17 (19.8\%), respectively; at 7-24 hours, the numbers halved to $15(16.9 \%)$ and $8(9.1 \%)$, respectively.

\section{Discussion}

Pain is one of the major complications after spine surgery. The cutoff points for mild, moderate, and severe pain in pain assessment are still unclear, and they are dependent on the patients' pain-thresholds $[15,16]$. To highlight the problem during the postoperative spine surgery period, the definition of severe pain employed in this study was an NRS of $\geq 7$, which provided an explicit cutoff point. The 
Table 2. Subgroup analysis factors related to postoperative pain intensity for the group of diagnoses related to high postoperative pain scores

\begin{tabular}{|c|c|c|c|}
\hline \multirow{2}{*}{ Variable } & \multicolumn{2}{|c|}{ Pain intensity in post-anesthesia care unit } & \multirow{2}{*}{$p$-value } \\
\hline & Mild-moderate pain (n=71) & Severe pain $(n=77)$ & \\
\hline Age group (yr) & & & 0.056 \\
\hline $0-18$ & 0 & 0 & \\
\hline $19-65$ & $38(53.5)$ & $53(68.8)$ & \\
\hline$>65$ & $33(46.5)$ & $24(31.2)$ & \\
\hline American Society of Anesthesia status & & & 0.223 \\
\hline I & $6(8.5)$ & $10(13.0)$ & \\
\hline$\|$ & $44(62.0)$ & $53(68.8)$ & \\
\hline III & $21(29.6)$ & $14(18.2)$ & \\
\hline Sex (female) & $40(56.3)$ & $47(61.0)$ & 0.562 \\
\hline Preoperative pain score (Visual Analog Scale) & $4.4 \pm 2.6$ & $4.7 \pm 2.5$ & 0.441 \\
\hline Baseline Oswestry Disability Index unit (missing 8 cases) & $42.2 \pm 20.6$ & $49.7 \pm 16.2$ & $0.018^{*}$ \\
\hline Chronic pain & $37(52.1)$ & $40(51.9)$ & 0.984 \\
\hline Level of laminectomy & & & 0.574 \\
\hline 0 & $11(15.5)$ & $13(16.9)$ & \\
\hline $1-2$ & $33(46.5)$ & $41(53.2)$ & \\
\hline$>2$ & $27(38.0)$ & $23(29.9)$ & \\
\hline Level of posterior instrument & & & 0.657 \\
\hline 0 & $5(7.0)$ & $10(13.0)$ & \\
\hline $1-2$ & $27(38.0)$ & $29(37.7)$ & \\
\hline $3-5$ & $34(47.9)$ & $34(44.2)$ & \\
\hline$>5$ & $5(7.0)$ & $4(5.2)$ & \\
\hline Level of interbody fusion & & & 0.360 \\
\hline 0 & $25(35.2)$ & $36(46.8)$ & \\
\hline 1 & $32(45.1)$ & $29(37.7)$ & \\
\hline$>2$ & $14(19.7)$ & $12(15.6)$ & \\
\hline Type of interbody fusion & & & 0.234 \\
\hline Posterior lumbar interbody fusion & $20(28.2)$ & $12(15.6)$ & \\
\hline Transforaminal lumbar interbody fusion & $15(21.1)$ & $19(24.7)$ & \\
\hline ALIF/XLIF/OLIF & $11(15.5)$ & $10(13.0)$ & \\
\hline None & $25(35.2)$ & $36(46.8)$ & \\
\hline Surgical duration (min) & $242 \pm 108$ & $204 \pm 76$ & $0.018^{*}$ \\
\hline Blood loss (mL) & $500(250-800)$ & $400(175-700)$ & 0.055 \\
\hline Nitrous oxide & $23(32.4)$ & $28(36.4)$ & 0.612 \\
\hline Minimum alveolar concentration of inhalation & $0.66 \pm 0.15$ & $0.68 \pm 0.16$ & 0.272 \\
\hline Morphine equivalence (mcg/kg/min) & $1.04 \pm 0.57$ & $1.89 \pm 0.61$ & 0.167 \\
\hline Local infiltration & 30 (42.3) & $34(44.2)$ & 0.815 \\
\hline Patient-controlled analgesia (opioids) & $25(35.2)$ & $15(19.5)$ & $0.031^{*}$ \\
\hline
\end{tabular}

Values are presented as number (\%), mean \pm standard deviation, or median (interquartile range).

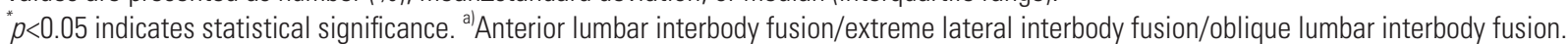


Table 3. Factors influencing severe pain during early postoperative period after multivariable analysis

\begin{tabular}{lcc} 
Variable & $p$-value & Adjusted odd ratio (95\% confidence interval) \\
Age (19-65 yr) & $0.007^{*}$ & $2.86(1.32-6.25)$ \\
Baseline Oswestry Disability Index unit & $0.004^{*}$ & $1.03(1.01-1.05)$ \\
Surgical duration (min) & 0.236 & $0.99(0.99-1.00)$ \\
Blood loss (mL) & 0.797 & $1.00(1.00-1.00)$ \\
Patient-controlled analgesia (opioids) & 0.059 & $0.44(0.19-1.03)$ \\
\hline
\end{tabular}

$p<0.05$ indicates statistical significance.

Table 4. Postoperative results

\begin{tabular}{|c|c|c|c|}
\hline \multirow{2}{*}{ Variable } & \multicolumn{2}{|c|}{ Pain intensity in PACU } & \multirow{2}{*}{$p$-value } \\
\hline & Mild-moderate pain (n=90) & Severe pain $(\mathrm{n}=90)$ & \\
\hline PACU duration (min) & $78.4 \pm 25.4$ & $86.2 \pm 27.3$ & $0.048^{*}$ \\
\hline Morphine in PACU (mg) & $0(0-0)$ & $4(2-6)$ & $<0.001^{*}$ \\
\hline Frequency of opioids received (times) & $0(0-0)$ & $2.5(1.75-3)$ & $<0.001^{*}$ \\
\hline Maximum NRS at $0-6 \mathrm{hr}$ & $4(0-6)$ & $5(3-7)$ & $0.002^{*}$ \\
\hline Maximum NRS at 7-24 hr & $2(0-5)$ & $4(2-5)$ & $0.006^{*}$ \\
\hline Severe pain at 0-6 hr (NRS) & $17(19.8)$ & $33(37.1)$ & $0.009^{*}$ \\
\hline Severe pain at 7-24 hr (NRS) & $8(9.1)$ & $15(16.9)$ & 0.094 \\
\hline
\end{tabular}

Values are presented as mean \pm standard deviation, median (interquartile range), or number (\%).

PACU, post-anesthesia care unit; NRS, Numerical Rating Scale.

* $p<0.05$ indicates statistical significance.

incidence of severe pain after thoracolumbar spine surgery in this study was $53.3 \%$, which is a matter of concern.

The results of the previously mentioned pilot study suggested that $\mathrm{N}_{2} \mathrm{O}$ may be associated with the development of severe pain during the early postoperative period. Nevertheless, the current study found no correlation between the intraoperative use of $\mathrm{N}_{2} \mathrm{O}$ and the early-postoperative severe pain. $\mathrm{N}_{2} \mathrm{O}$ was used as an adjuvant anesthetic gas in $59(32.78 \%)$ of the patients undergoing thoracolumbar spine surgery. Although the concentration of inhaled anesthetic was noticeably reduced with statistical significance in the $\mathrm{N}_{2} \mathrm{O}$ subgroup (minimum alveolar concentration of inhalation was $0.61 \pm 0.16$ versus $0.72 \pm 0.14$ in the non- $\mathrm{N}_{2} \mathrm{O}$ subgroup; $p<0.001$ ), the use of $\mathrm{N}_{2} \mathrm{O}$ did not yield any clinical significance. Furthermore, a decrement in the intraoperative total opioid doses was not observed in the current study. These results suggest that the advantages of $\mathrm{N}_{2} \mathrm{O}$ for spine surgery are still indistinct, and, therefore, its use is still debatable. Consequently, before adopting $\mathrm{N}_{2} \mathrm{O}$ for use in clinical practice, it is important to weigh the perceived benefits against the risks.
The disadvantages of employing $\mathrm{N}_{2} \mathrm{O}$ for spine surgery include the risk of pneumocephalus or air entrapment in the epidural space, which may result in subsequent neurological deficits in patients whose dura is torn or intentionally opened $[17,18]$. Moreover, the use of $\mathrm{N}_{2} \mathrm{O}$ increases the risk for postoperative nausea and vomiting. However, postoperative cognitive dysfunction and cardiovascular complications are not associated with $\mathrm{N}_{2} \mathrm{O}$, according to the study of Myles et al. [19].

The surgical diagnoses which related to severe pain were FBSS, spinal stenosis, and spondylolisthesis. The diagnoses of adolescent idiopathic scoliosis, fracture, cancer, infection, and the rest seemed to have lower pain scores during the early postoperative period. However, surgical diagnosis was not noted to be a risk factor for postoperative severe pain in the PACU, as demonstrated in Table 1. From the subgroup analysis of the diagnoses related to high pain scores, it was found that a patient aged 19-65 years and/or with a high baseline ODI score was at significant risk for early postoperative severe pain in the PACU.

Depression, the preoperative pain score, and chronic 
preoperative opioid use had been reported as important risk factors for postoperative pain and other complications $[4,8,9]$. However, the analysis of the patients' psychological profiles or mental states was not routine at our institute. The preoperative spinal surgery evaluation protocol involved the preoperative pain (VAS) and baseline ODI scores, except for patients with diagnosed scoliosis. According to a medical record review, only four patients were taking antidepressant medications (amitriptyline or nortriptyline) and 92 patients (51\%) were taking gabapentin, pregabalin, or tramadol, presumably due to their chronic pain. No prescriptions for preoperative strong opioids were noted. Because depression screening has been an ongoing issue in Thailand, our study had a limited ability to explore the factors related to mental status. Nonetheless, this study found that a high baseline ODI score may increase the risk of severe pain after thoracolumbar spine surgery, albeit further studies are needed to establish the relevant cutoff point. Unlike in previous studies, the preoperative pain score failed to be a statistically significant risk factor in our study. We thus propose that a single preoperative pain score cannot be a reliable assessment for spinal surgery patients.

In this study, the postoperative use of IV PCA seemed to be a preventive factor that improved the pain outcomes during the early postoperative period, specifically until the end of the first 24 hours after surgery (adjusted OR, 0.05). However, IV PCA was not a significant preventive factor in the subgroup of FBSS, spinal stenosis, and spondylolisthesis. Patients who suffered from severe pain showed a tendency toward a longer PACU stay since having adequately controlled pain is mandatory prior to PACU discharge (Table 4). Although the standardized postoperative pain protocol is presently inconclusive for major spine surgeries, IV PCA-opioids seem to be are a promising option for postoperative pain control with minimal adverse effects. Other satisfactory alternatives for pain control after major spine surgery are a single-shot or continuous epidural analgesia and intrathecal morphine. These techniques can reduce the IV opioid consumption; nonetheless, several studies have reported that pruritus and postoperative nausea vomiting were more common with these alternatives [5,20,21].

Several published studies have suggested that preoperative pain intensity is a major predictor for the occurrence of postoperative severe pain [4,22]. An individual postoperative pain control regimen can possibly be established with the continuous evaluation of a patient's perioperative pain intensity and perception. The utilization of multiphasic and multimodal analgesia is strongly recommended $[23,24]$. The current study cannot substantiate the result of preoperative pain intensity toward the development of severe pain because a systematic evaluation of the preoperative pain score is insufficient. Although the preoperative use of chronic pain medications may be suggestive, the duration of the pain is still lacking.

Since the development of postoperative intense pain after major spine surgery is difficult to control, the establishment of a postoperative pain control protocol for major spine surgery is crucial to relieve postoperative pain to the maximum possible extent. If the protocol was available, the patients would be able to promptly recover from surgery, and the postoperative complications would be significantly reduced. Enhanced recovery after surgery should also be advocated with a multidisciplinary team-namely surgeons, anesthesiologists, nurses, and physiotherapists-who should be present throughout the perioperative period to help achieve the best outcomes for patients undergoing major spine surgeries [25-28].

Since the study was designed as a retrospective chart review, some data were not completely recorded, such as the psychological profile, the diagnosis of chronic pain, the preoperative pain intensity, the local anesthetic or ketorolac infiltration by the surgeons, and the delivered dose of opioids via a PCA machine. Our findings are also subject to bias from unmeasured confounders such as surgeons, anesthesiologists, and PACU nurses who assessed the pain intensity.

\section{Conclusions}

The anesthetic regimen of general anesthesia for thoracolumbar spine surgery and surgical techniques did not affect the early postoperative severe pain. An age under 66 years and a high baseline ODI were the significant risk factors for pain intensity during the early postoperative period in cases of FBSS, spinal stenosis, and spondylolisthesis.

\section{Conflict of Interest}

No potential conflict of interest relevant to this article was reported. 


\section{Acknowledgments}

The authors are indebted to the Siriraj R2R team members for consultation on this paper; Miss Julaporn Pooliam for assistance with the methodology; Miss Chusana Rungjindamai for her invaluable support with administrative work; and Mr David Park for thoroughly copy editing the manuscript.

\section{Funding}

This research project was supported by Siriraj Research Development Fund (managed by Routine to Research: R2R), Faculty of Medicine Siriraj Hospital, Mahidol University (grant no., 17AS00024/003/17).

\section{ORCID}

Paweenus Rungwattanakit: https://orcid.org/0000-0002-2502-9534 Tarnkamon Sondtiruk: https://orcid.org/0000-0002-2227-0795 Akarin Nimmannit: https://orcid.org/0000-0001-7937-2306 Busara Sirivanasandha: https://orcid.org/0000-0002-8821-5043

\section{Author Contributions}

Conception and design: PR, TS, BS; acquisition and data: PR, TS; analysis and interpretation of data: PR, TS, BS; drafting of the manuscript: BS; critical revision of the manuscript for important intellectual content: AN, BS; statistical analysis: AN, BS; supervision: AN, BS; obtaining funding: BS; and administrative, technical, or material support: BS

\section{References}

1. Thienthong S, Jirarattanaphochai K, Krisanaprakornkit W, Simajareuk S, Tantanatewin W, Sathitkarnmanee A. Treatment of pain after spinal surgery in the recovery room by single dose lornoxicam: a randomized, double blind, placebo-controlled trial. J Med Assoc Thai 2004;87:650-5.

2. Nielsen RV, Fomsgaard JS, Dahl JB, Mathiesen O. Insufficient pain management after spine surgery. Dan Med J 2014;61:A4835.

3. Gerbershagen HJ, Aduckathil S, van Wijck AJ, Peelen LM, Kalkman CJ, Meissner W. Pain intensity on the first day after surgery: a prospective cohort study comparing 179 surgical procedures. Anesthesiology 2013;118:934-44.

4. Kim HJ, Park JH, Kim JW, et al. Prediction of postoperative pain intensity after lumbar spinal surgery using pain sensitivity and preoperative back pain severity. Pain Med 2014;15:2037-45.

5. Wu MH, Wong CH, Niu CC, Tsai TT, Chen LH, Chen WJ. A comparison of three types of postoperative pain control after posterior lumbar spinal surgery. Spine 2011;36:2224-31.

6. Brown CH 4th, LaFlam A, Max L, et al. Delirium after spine surgery in older adults: incidence, risk factors, and outcomes. J Am Geriatr Soc 2016;64:21018.

7. Ganter MT, Blumenthal S, Dubendorfer S, et al. The length of stay in the post-anaesthesia care unit correlates with pain intensity, nausea and vomiting on arrival. Perioper Med 2014;3:10.

8. McKillop AB, Carroll LJ, Battie MC. Depression as a prognostic factor of lumbar spinal stenosis: a systematic review. Spine J 2014;14:837-46.

9. Dunn LK, Yerra S, Fang S, et al. Incidence and risk factors for chronic postoperative opioid use after major spine surgery: a cross-sectional study with longitudinal outcome. Anesth Analg 2018;127:247-54.

10. Charuluxananan $S$, Thienthong $S$, Rungreungvanich M, Srirojanakul W, Punjasawadwong Y, Sriprajittichai P. A survey of post anesthetic pain management in Thailand. J Med Assoc Thai 2009;92:1028-32.

11. Urban MK, Jules-Elysee K, Urquhart B, Cammisa FP, Boachie-Adjei O. Reduction in postoperative pain after spinal fusion with instrumentation using intrathecal morphine. Spine 2002;27:535-7.

12. Emmanouil DE, Quock RM. Advances in understanding the actions of nitrous oxide. Anesth Prog 2007;54:9-18.

13. Ben Boujema M, Laboureyras E, Pype J, Bessiere B, Simonnet G. Nitrous oxide persistently alleviates pain hypersensitivity in neuropathic rats: a dosedependent effect. Pain Res Manag 2015;20:309-15.

14. Duma A, Helsten D, Brown F, Bottros MM, Nagele P. The effect of nitrous oxide anesthesia on early postoperative opioid consumption and pain. Reg Anesth Pain Med 2014;39:31-6.

15. Boonstra AM, Stewart RE, Koke AJ, et al. Cut-off points for mild, moderate, and severe pain on the numeric rating scale for pain in patients with chronic 
musculoskeletal pain: variability and influence of sex and catastrophizing. Front Psychol 2016;7:1466.

16. Gerbershagen HJ, Rothaug J, Kalkman CJ, Meissner W. Determination of moderate-to-severe postoperative pain on the numeric rating scale: a cut-off point analysis applying four different methods. Br J Anaesth 2011;107:619-26.

17. Theilen HJ, Heller AR, Litz RJ. Nitrous oxide-induced tension pneumocephalus after thoracic spinal cord surgery: a case report. J Neurosurg Anesthesiol 2008;20:211-2.

18. Kaymaz M, Oztanir N, Emmez H, Ozkose Z, Paşaoglu A. Epidural air entrapment after spinal surgery. Clin Neurol Neurosurg 2005;107:421-4.

19. Myles PS, Leslie K, Chan MT, et al. The safety of addition of nitrous oxide to general anaesthesia in at-risk patients having major non-cardiac surgery (ENIGMA-II): a randomised, single-blind trial. Lancet 2014;384:1446-54.

20. Duger C, Gursoy S, Karadag O, et al. Anesthetic and analgesic effects in patients undergoing a lumbar laminectomy of spinal, epidural or a combined spinal-epidural block with the addition of morphine. J Clin Neurosci 2012;19:406-10.

21. Cohen M, Zuk J, McKay N, Erickson M, Pan Z, Galinkin J. Intrathecal morphine versus extendedrelease epidural morphine for postoperative pain control in pediatric patients undergoing posterior spinal fusion. Anesth Analg 2017;124:2030-7.

22. Gu J, Guan F, Zhu L, et al. Risk factors of postoperative low back pain for lumbar spine disease. World Neurosurg 2016;94:248-54.

23. Kurd MF, Kreitz T, Schroeder G, Vaccaro AR. The role of multimodal analgesia in spine surgery. J Am Acad Orthop Surg 2017;25:260-8.

24. Devin CJ, McGirt MJ. Best evidence in multimodal pain management in spine surgery and means of assessing postoperative pain and functional outcomes. J Clin Neurosci 2015;22:930-8.

25. Ali ZS, Ma TS, Ozturk AK, et al. Pre-optimization of spinal surgery patients: development of a neurosurgical enhanced recovery after surgery (ERAS) protocol. Clin Neurol Neurosurg 2018;164:142-53.

26. Wainwright TW, Immins T, Middleton RG. Enhanced recovery after surgery (ERAS) and its applicability for major spine surgery. Best Pract Res Clin Anaesthesiol 2016;30:91-102.

27. Development of an Enhanced Recovery After Surgery (ERAS) approach for lumbar spinal fusion. J Neurosurg Spine 2017;26:411-8.

28. Nielsen PR, Jorgensen LD, Dahl B, Pedersen T, Tonnesen H. Prehabilitation and early rehabilitation after spinal surgery: randomized clinical trial. Clin Rehabil 2010;24:137-48. 